\title{
Non-Data Aided Feedforward Estimation of PSK-Modulated Carrier Frequency Offset
}

\author{
Y. Wang ${ }^{1}$, E. Serpedin ${ }^{1}$ and P. Ciblat ${ }^{2}$ \\ ${ }^{1}$ Dept. of Electrical Engineering, Texas A\&M University, College Station, TX 77843, USA \\ ${ }^{2}$ Ecole Nationale Supérieure des Télécommunications, Paris, France
}

\begin{abstract}
This paper examines the asymptotic (large sample) performance of a family of non-data aided feedforward (NDA FF) nonlinear least-squares (NLS) type carrier frequency estimators for burst-mode phase shift keying (PSK) modulations transmitted through AWGN and flat Ricean-fading channels. The asymptotic performance of these estimators is established in closed-form expression and compared with the modified Cramèr-Rao bound (MCRB). A best linear unbiased estimator (BLUE), which exhibits the lowest asymptotic variance within the family of NDA FF NLS-type estimators, is also proposed.
\end{abstract}

\section{INTRODUCTION}

Burst transmission of digital data and voice is employed in time division-multiple access (TDMA) and packet demandassignment multiple-access (DAMA) satellite communication and terrestrial mobile cellular radio systems. Conventionally, synchronization of burst transmissions requires a large number of overhead symbols, which results in reduced spectral efficiency and increased transmission delays [4].

In this paper, a family of non-data aided (NDA) or blind nonlinear least-squares (NLS) feedforward frequency offset estimators for carriers that are fully modulated by M-ary phase shift keying (PSK) modulations is proposed and its asymptotic (large sample) performance analyzed in a rigorous way. The proposed frequency offset estimators exploit a generalized form of the Maximum Likelihood Feedforward (ML FF) algorithm, that was originally proposed by A. J. Viterbi and A. M. Viterbi as a blind carrier phase estimator with improved performance at low and intermediate Signal-to-Noise Ratios (SNRs) [12], [10]. This carrier phase estimator is referred in the literature as the Viterbi and Viterbi (V\&V) algorithm [5], $[9$, p. 280].

A number of blind frequency offset estimators for burst M-PSK modulations were reported and partially analyzed for AWGN channels, without taking into account possible fading effects [2], [3]. Reference [5] introduces another family of non-data aided feedforward carrier frequency offset estimators that rely on a rather restrictive set of assumptions and whose asymptotic variance is evaluated based on several approximations. The goal of the present paper is to examine rigorously the performance of the blind least-squares estimator [8] in AWGN and flat Ricean-fading channels, and to propose estimators with improved performance.

As we shall see, the proposed family of nonlinear frequency offset estimators presents high convergence rates (asymptotic variances on the order of $\mathrm{O}\left(1 / N^{3}\right)$, where $N$ denotes the number of available samples), provides high accurate frequency offset estimates, can cope with large frequency offsets ( $\pm(50 / M) \%$ of the symbol rate), and admits low complexity digital implementations, without being necessary to oversample (fractionally-sample) the received signal faster than the Nyquist rate. The performance of these algorithms achieves closely the modified Cramèr-Rao bound (MCRB) at medium and high SNRs, and is robust to Ricean fading effects and timing errors.

\section{PROBLEM FORMULATION}

Consider the baseband representation of an M-PSK modulated signal transmitted through an AWGN channel. Assume that filtering is evenly split between transmitter and receiver so that the overall channel is Nyquist. Filtering the received waveform through a matched filter and sampling at the right time instants yields:

$$
x(n)=w(n) e^{j\left(2 \pi F_{e} T n+\theta\right)}+v(n), n=0,1, \ldots, N-1,
$$

where $\{w(n)\}$ is the sequence of zero-mean unit variance $\left(\sigma_{w}^{2}:=\mathrm{E}\left\{|w(n)|^{2}\right\}=1\right)$ independently and identically distributed (i.i.d.) M-PSK symbols, $\theta$ and $f_{e}:=F_{e} T$ stand for carrier phase and frequency offset, respectively, $T$ denotes the symbol period, and $\{v(n)\}$ is a zero-mean white Gaussian noise process independent of $w(n)$ and with variance $\sigma_{v}^{2}:=\mathrm{E}\left\{|v(n)|^{2}\right\}$. The Signal-to-Noise Ratio is defined as SNR: $=10 \log _{10}\left(\sigma_{w}^{2} / \sigma_{v}^{2}\right)$. Furthermore, without any loss in generality, we assume for the rest of the paper that $\theta=0$. Note that a non-zero value for $\theta$ is immaterial to the analysis that will be carried over in this paper. In addition, $\theta$ can be estimated by means of the $\mathrm{V} \& \mathrm{~V}$ estimator [12] after the frequency offset is determined.

As depicted by (1), the problem that we pose is to estimate the unknown frequency $f_{e}$ of a harmonic embedded in unknown multiplicative $(w(n))$ and additive noise $(v(n))$, assuming knowledge of the received samples $\{x(n)\}_{n=0}^{N-1}$. The solution that we pursue consists of evaluating first certain moments of the output that will remove the unwanted multiplicative effects introduced by the M-PSK modulated sequence $w(n)$. It turns out that the resulting problem reduces to the standard problem of estimating the frequency of a constant amplitude harmonic embedded in additive noise, for which standard NLStype estimators can be developed and their statistical properties analyzed in a rigorous manner.

\section{FREQUENCY OFFSET ESTIMATION}

Consider the polar representation:

$$
x(n)=\rho(n) e^{j \varphi(n)},
$$

and define the family of processes:

$$
y_{k}(n)=\rho^{k}(n) e^{j M \varphi(n)}, k=0,1, \ldots, M .
$$

For $k=0, \ldots, M$, introduce the class of frequency estimators:

$$
\hat{f}_{e}^{(k)}=\frac{1}{M} \arg \max _{\left|\overline{f_{0}}\right|<1 / 2}\left|\frac{1}{N} \sum_{n=0}^{N-1} y_{k}(n) e^{-j 2 \pi \overline{f_{0}} n}\right| .
$$


In order to establish the asymptotic unbiasedness and consistency of estimators (4), some preparatory results are reviewed next. Conditioned on $w(n), x(n)$ is normally distributed with the probability density function (pdf) $f(x(n) \mid w(n)=$ $2 \pi l / M, 0 \leq l \leq M-1) \sim \mathcal{N}\left(w(n) \exp \left(j 2 \pi f_{e} n\right), \sigma_{v}^{2}\right)$. Due to (2), it follows that:

$$
\begin{aligned}
f(\rho(n), \varphi(n) \mid w(n) & =2 \pi l / M)=\frac{\rho(n)}{\pi \sigma_{v}^{2}} e^{-\left(\rho^{2}(n)+1\right) / \sigma_{v}^{2}} \\
\cdot & e^{2 \rho(n) \cos \left[\varphi(n)-2 \pi\left(l+M f_{e} n\right) / M\right] / \sigma_{v}^{2}} .
\end{aligned}
$$

Based on (5), the joint and marginal pdf of $\rho(n)$ and $\varphi(n)$ take the expressions:

$$
\begin{gathered}
f(\rho(n), \varphi(n))=\frac{1}{M} \sum_{l=0}^{M-1} f\left(\rho(n), \varphi(n) \mid w(n)=\frac{2 \pi l}{M}\right) \\
=\frac{1}{M} \sum_{l=0}^{M-1} \frac{\rho(n)}{\pi \sigma_{v}^{2}} e^{-\frac{\rho^{2}(n)+1}{\sigma_{v}^{2}}} e^{\frac{2 \rho(n)}{\sigma_{v}^{2}} \cos \left(\varphi(n)-\frac{2 \pi\left(l+M f_{e} n\right)}{M}\right)}, \\
f(\rho(n))=\int_{-\pi}^{\pi} f(\rho(n), \varphi(n)) \mathrm{d} \varphi(n) \\
=\frac{2 \rho(n)}{\sigma_{v}^{2}} e^{-\left(\rho^{2}(n)+1\right) / \sigma_{v}^{2}} I_{0}\left(\frac{2 \rho(n)}{\sigma_{v}^{2}}\right),
\end{gathered}
$$

where $I_{0}(\cdot)$ stands for the zero-order modified Bessel function of the first kind [1, eq. (9.6.16)]. Using (6), some direct calculations show that:

$$
\mathrm{E}\left\{y_{k}(n)\right\}=\frac{1}{\alpha^{k}} e^{j 2 \pi M f_{e} n} e^{-\frac{\gamma}{2}} \int_{0}^{\infty} \zeta^{k+1} e^{-\frac{\zeta^{2}}{2}} I_{M}(\alpha \zeta) \mathrm{d} \zeta,
$$

where: $\alpha:=\sqrt{2} / \sigma_{v}, \gamma:=\alpha^{2}, \zeta:=\alpha \rho(n)$ and $I_{M}(\cdot)$ denotes the M-order modified Bessel function of the first kind [1, eq. (9.6.19)]. Based on [7, eq. (6.643,2)] and [1, eq. (13.1.32)], the RHS of (8) can be expressed in terms of the confluent hypergeometric function $\Phi(\cdot, \cdot, \cdot)$ :

$$
\begin{aligned}
& \mathrm{E}\left\{y_{k}(n)\right\}=A_{k} e^{j 2 \pi M f_{e} n}, k=0,1, \ldots, M, \\
& A_{k}:=\frac{\Gamma\left(\frac{k+M}{2}+1\right) e^{-\frac{\gamma}{2}}}{\Gamma(M+1) \sigma_{v}^{M-k}} \Phi\left(\frac{k+M}{2}+1, M+1, \frac{\gamma}{2}\right) .
\end{aligned}
$$

Since $w(n)$ and $v(n)$ are i.i.d. and mutually independent, it follows that $v_{k}(n):=y_{k}(n)-\mathrm{E}\left\{y_{k}(n)\right\}$ is i.i.d., too. Consequently,

$$
y_{k}(n)=A_{k} e^{j 2 \pi M f_{e} n}+v_{k}(n), n=0,1, \ldots, N-1,
$$

and $y_{k}(n)$ can be viewed as a constant amplitude harmonic embedded in white noise. Notice that in general, $v_{k}(n)$ is not circular. Due to well-known properties of NLS-estimators, it follows immediately that estimator (4) is asymptotically unbiased and consistent [11, p. 147]. Identifiability of $f_{e}$ in (4) requires that $\left|2 \pi M f_{e}\right|<\pi$, which implies $\left|F_{e}\right|<1 /(2 M T)$. Hence, estimators (4) can cope with relatively large frequency offsets $( \pm(50 / M) \%$ of the symbol rate). Following the procedures of [6], one can derive the asymptotic variance of (4) as:

$$
\operatorname{avar}\left(\hat{f}_{e}^{(k)}\right):=\lim _{N \rightarrow \infty} N^{3} \mathrm{E}\left\{\left(\hat{f}_{e}^{(k)}-f_{e}\right)^{2}\right\}=\frac{6\left(B_{k}-C_{k}\right)}{4 \pi^{2} M^{2}\left|A_{k}\right|^{2}},
$$

where:

$$
\begin{aligned}
& B_{k}:=\mathrm{E}\left\{\left|y_{k}(n)\right|^{2}\right\}=\mathrm{E}\left\{\rho^{2 k}(n)\right\} \\
& C_{k}:=\left|\mathrm{E}\left\{y_{k}^{2}(n)\right\}\right|=\left|\mathrm{E}\left\{\rho^{2 k}(n) e^{j 2 M \varphi(n)}\right\}\right|
\end{aligned}
$$

Exploiting (7) and [7, eq. (6.643.4)], the following relation was derived in $[12,(\mathrm{~A} 17)]$ :

$$
\mathrm{E}\left\{\rho^{2 k}(n)\right\}=\sigma_{v}^{2 k} \sum_{q=0}^{k}\left(\begin{array}{l}
k \\
q
\end{array}\right)^{2}\left(\frac{\gamma}{2}\right)^{k-q} \cdot q ! .
$$

Similar to (10), $C_{k}$ can be obtained as:

$$
C_{k}=\frac{\Gamma(k+M+1) e^{-\frac{\gamma}{2}}}{\Gamma(2 M+1) \sigma_{v}^{2 M-2 k}} \Phi\left(k+M+1,2 M+1, \frac{\gamma}{2}\right) .
$$

Following a similar approach to that presented in [12], one can obtain a slightly more compact expression for the confluent hypergeometric function in (14):

$$
\begin{aligned}
C_{k}= & \frac{1}{\gamma^{k}}\left[\gamma^{k} \sum_{p=0}^{M+k} p !\left(\begin{array}{c}
M+k \\
p
\end{array}\right)\left(\begin{array}{c}
M-k+p-1 \\
p
\end{array}\right)\left(\frac{-2}{\gamma}\right)^{p}\right. \\
& +(-1)^{M+k+1} 2^{k} e^{-\frac{\gamma}{2}}\left(\frac{2}{\gamma}\right)^{k+1} \sum_{p=0}^{M-k-1}\left(\begin{array}{c}
M+k+p \\
p
\end{array}\right) \\
& \left.\cdot \frac{(M+k) !}{(M-k-p-1) !}\left(\frac{2}{\gamma}\right)^{p}\right], \text { if } k=0,1, \ldots, M-1,
\end{aligned}
$$

$C_{k}=1$, if $k=M$.

Plugging (10), (13) and (15) back into (12), a closed-form expression for the asymptotic variance $\operatorname{avar}\left(\hat{f}_{e}^{(k)}\right)$ is obtained.

Now it is interesting to compare the asymptotic variances (12) corresponding to different estimator orders $k$. First, we will study the high $\mathrm{SNR}$ asymptotic regime $(\mathrm{SNR} \rightarrow \infty)$. Using [1, eq. (13.1.4)], some direct calculations show that:

$$
\lim _{\mathrm{SNR} \rightarrow \infty} A_{k}=1
$$

for any $k=0,1, \ldots, M$. Hence, based on (13) and (15) we obtain:

$$
\lim _{\mathrm{SNR} \rightarrow \infty} \operatorname{avar}\left(\hat{f}_{e}^{(k)}\right)=\frac{6}{4 \pi^{2}} \cdot \frac{1}{\mathrm{SNR}}+O\left(\frac{1}{\mathrm{SNR}^{2}}\right) .
$$

Consequently,

$$
\lim _{\mathrm{SNR} \rightarrow \infty} \frac{\operatorname{avar}\left(\hat{f}_{e}^{(k)}\right)}{\operatorname{avar}\left(\hat{f}_{e}^{(M)}\right)}=1+O\left(\frac{1}{\mathrm{SNR}}\right),
$$

which shows that at high SNR asymptotic regime, the performance of estimators (4) for different orders $k$ is asymptotically the same. The study of the asymptotic low SNR behavior of estimators (4) requires evaluating confluent hypergeometric functions in terms of the power series expansion [1, eq. (13.1.2)]. From the plots depicted in Figs. 2-3, it turns out that the best performance in the low SNR range is achieved for $k=1$ and 2 
in the case of envisaged M-PSK constellations ( $M=2,4$ and 8 ). It should be pointed out that when $k$ is even ( $M$ is usually a power of two), similar to (15), one can obtain:

$$
\begin{aligned}
A_{k}= & \frac{1}{\gamma^{t}}\left[\gamma^{t} \sum_{p=0}^{s+t} p !\left(\begin{array}{c}
s+t \\
p
\end{array}\right)\left(\begin{array}{c}
s-t+p-1 \\
p
\end{array}\right)\left(\frac{-2}{\gamma}\right)^{p}\right. \\
& +(-1)^{s+t+1} 2^{t} e^{-\frac{\gamma}{2}}\left(\frac{2}{\gamma}\right)^{t+1} \sum_{p=0}^{s-t-1}\left(\begin{array}{c}
s+t+p \\
p
\end{array}\right) \\
& \left.\cdot \frac{(s+t) !}{(s-t-p-1) !}\left(\frac{2}{\gamma}\right)^{p}\right], \text { if } k=0,2, \ldots, M-2, \\
A_{k}= & 1, \text { if } k=M,
\end{aligned}
$$

where $s:=M / 2$ and $t:=k / 2$.

Next, we determine the best linear unbiased estimator (BLUE) that achieves the lowest asymptotic variance within the class of all moment estimators (4). Define the estimator:

$$
\begin{aligned}
& \hat{f}_{e}^{(l i n)}=\frac{1}{M} \arg \max _{\left|\overline{f_{0}}\right|<1 / 2}\left|\frac{1}{N} \sum_{n=0}^{N-1} g(n) e^{-j 2 \pi \bar{f}_{0} n}\right|, \\
& g(n):=\sum_{k=0}^{M} g_{k} y_{k}(n)=\left(\underline{\underline{o}}^{T}(n) \cdot \mathbf{g}\right) e^{j M \varphi(n)}, \forall n,
\end{aligned}
$$

where $g_{k}, k=0,1, \ldots, M$, are some scalars that will be chosen to minimize the asymptotic variance of $\hat{f}_{e}^{(l i n)}$ in (19), and:

$$
\begin{aligned}
& \mathbf{g}:=\left[\begin{array}{llll}
g_{0} & g_{1} & \ldots & g_{M}
\end{array}\right]^{T},
\end{aligned}
$$

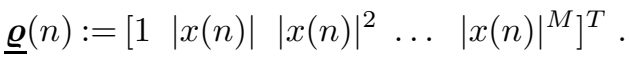

Using (20) and (9), we obtain that:

$$
\mathrm{E}\{g(n)\}:=\left(\sum_{k=0}^{M} g_{k} A_{k}\right) e^{j 2 \pi M f_{e} n}=\left(\underline{\mathbf{a}}^{T} \mathbf{g}\right) e^{j 2 \pi M f_{e} n},
$$

where $\underline{\mathbf{a}}:=\left[\begin{array}{llll}A_{0} & A_{1} & \ldots & A_{M}\end{array}\right]^{T}$. Since $w(n)$ and $v(n)$ are i.i.d. and mutually independent, it follows that $v^{(\operatorname{lin})}(n):=$ $g(n)-\mathrm{E}\{g(n)\}$ is also a white noise sequence. We have also:

$$
\mathrm{E}\left\{|g(n)|^{2}\right\}=\underline{\mathbf{g}}^{T} \cdot \underline{\mathbf{R}} \cdot \mathbf{g}, \quad\left|\mathrm{E}\left\{g^{2}(n)\right\}\right|=\underline{\mathbf{g}}^{T} \cdot \underline{\tilde{\mathbf{R}}} \cdot \mathbf{g},
$$

with $\underline{\mathbf{R}}:=\mathrm{E}\left\{\underline{\underline{\boldsymbol{g}}}(n) \underline{\underline{\boldsymbol{\rho}}}^{T}(n)\right\}$ and $[\underline{\tilde{\mathbf{R}}}]_{k, l=0,1, \ldots, M}:=$ $\left|\mathrm{E}\left\{\rho^{k+l}(n) \exp (j 2 \bar{M} \varphi(n))\right\}\right|$. Exploiting (21) and (22), we obtain the asymptotic variance of estimator (19), which can be expressed as:

$$
\begin{aligned}
\operatorname{avar}\left(\hat{f}_{e}^{(l i n)}\right):= & \lim _{N \rightarrow \infty} N^{3} \mathrm{E}\left\{\left(\hat{f}_{e}^{(l i n)}-f_{e}\right)^{2}\right\} \\
& =\frac{6}{4 \pi^{2} M^{2}}\left(\frac{\mathbf{g}^{T} \cdot(\underline{\mathbf{R}}-\underline{\tilde{\mathbf{R}}}) \cdot \mathbf{g}}{\left|\mathbf{g}^{T} \cdot \underline{\mathbf{a}}\right|^{2}}\right) .
\end{aligned}
$$

Hence, finding BLUE resorts to solving the optimization problem:

$$
\hat{\mathbf{g}}^{(\mathrm{BLUE})}=\arg \min _{\mathbf{g} \neq \underline{\mathbf{0}}} \frac{\mathbf{g}^{T} \cdot(\underline{\mathbf{R}}-\underline{\tilde{\mathbf{R}}}) \cdot \underline{\mathbf{g}}}{\left|\mathbf{g}^{T} \cdot \underline{\mathbf{a}}\right|^{2}},
$$

whose standard solution is given by (c.f. [11, ch. 5]):

$$
\hat{\mathbf{g}}^{(\mathrm{BLUE})}=(\underline{\mathbf{R}}-\underline{\tilde{\mathbf{R}}})^{-1} \cdot \underline{\mathbf{a}} .
$$

Plugging (25) back into (23), we obtain:

$$
\operatorname{avar}\left(\hat{f}_{e}^{(\mathrm{BLUE})}\right)=\frac{6}{4 \pi^{2} M^{2}}\left(\frac{1}{\underline{\mathbf{a}}^{T} \cdot(\underline{\mathbf{R}}-\underline{\tilde{\mathbf{R}}})^{-1} \cdot \underline{\mathbf{a}}}\right) .
$$

Fig. 1 plots the loss in performance of estimators (4) w.r.t. $\operatorname{BLUE}(19)\left(-10 \log _{10}\left[\operatorname{avar}\left(\hat{f}_{e}^{(k)}\right) / \operatorname{avar}\left(\hat{f}_{e}^{(\mathrm{BLUE})}\right)\right]\right)$ in the case of a BPSK modulation $(M=2)$.

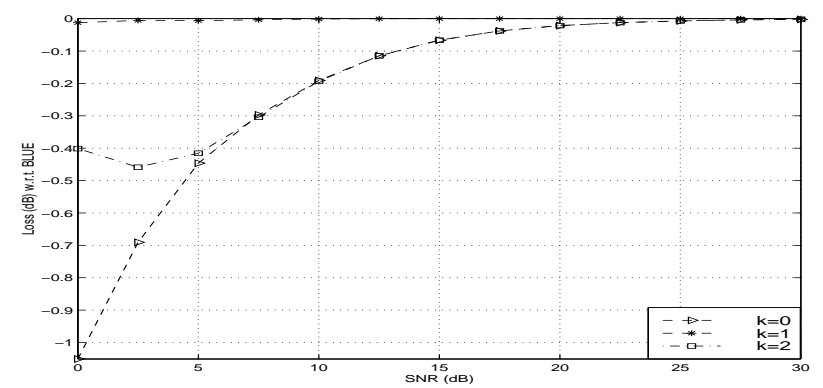

Fig. 1. Theoretical degradation of $\hat{f}_{e}^{(k)}$ w.r.t. $\hat{f}_{e}^{(\mathrm{BLUE})}$ versus SNR for BPSK constellation.

\section{EXTENSION TO FLAT RICEAN-FADING CHANNELS}

In the foregoing discussion, we assumed AWGN channels. In this section, we will see that the frequency offset estimators (4) remain unbiased and consistent in the presence of flat Ricean-fading channels.

When the channel is flat Ricean-fading channel, (1) will be modified to:

$$
x(n)=\mu(n) w(n) e^{j\left(2 \pi F_{e} T n+\theta\right)}+v(n), \forall n,
$$

where $\mu(n)=\rho_{\mu}(n) \exp \left(j \varphi_{\mu}(n)\right)$ is the fading process with non-zero mean $\operatorname{E}\{\mu(n)\}:=\rho_{1} \exp \left(j \varphi_{1}\right)$ and variance $\sigma_{\mu}^{2}:=\mathrm{E}\left\{|\mu(n)-\mathrm{E}\{\mu(n)\}|^{2}\right\}$. Using the Jakes model, we have: $\mathrm{E}\left\{[\mu(n)-\mathrm{E}\{\mu(n)\}]^{*}[\mu(n+\tau)-\mathrm{E}\{\mu(n+\tau)\}]\right\}=$ $\sigma_{\mu}^{2} J_{0}\left(2 \pi f_{d} \tau\right)$, where $J_{0}($.$) denotes the zero-order Bessel func-$ tion of the first kind, and $f_{d}$ is the normalized Doppler spread. The joint and marginal pdf of $\rho_{\mu}(n)$ and $\varphi_{\mu}(n)$ are given by:

$$
\begin{aligned}
& f\left(\rho_{\mu}(n), \varphi_{\mu}(n)\right)=\frac{\rho_{\mu}(n)}{\pi \sigma_{\mu}^{2}} e^{-\frac{\rho_{\mu}^{2}(n)+\rho_{1}^{2}-2 \rho_{\mu}(n) \rho_{1} \cos \left(\varphi_{\mu}(n)-\varphi_{1}\right)}{\sigma_{\mu}^{2}}} \\
& f\left(\rho_{\mu}(n)\right)=\frac{2 \rho_{\mu}(n)}{\sigma_{\mu}^{2}} e^{-\frac{\rho_{\mu}^{2}(n)+\rho_{1}^{2}}{\sigma_{\mu}^{2}}} I_{0}\left(\frac{2 \rho_{\mu}(n) \rho_{1}}{\sigma_{\mu}^{2}}\right)
\end{aligned}
$$

Conditioned on the fading process $\mu(n)$ and the input symbol $w(n)$, the joint pdf of $\rho(n)$ and $\varphi(n)$ takes the form:

$$
\begin{gathered}
f\left(\rho(n), \varphi(n) \mid w(n), \rho_{\mu}(n), \varphi_{\mu}(n)\right)=\frac{\rho(n)}{\pi \sigma_{v}^{2}} e^{-\left(\rho^{2}(n)+\rho_{\mu}^{2}(n)\right) / \sigma_{v}^{2}} \\
\cdot e^{2 \rho(n) \rho_{\mu}(n) \cos \left[\varphi(n)-\varphi_{\mu}(n)-2 \pi\left(l+M f_{e} n\right) / M\right] / \sigma_{v}^{2}} .
\end{gathered}
$$


Using (27) to (30), in a similar way to that presented in the last section, some straightforward but lengthy calculations lead to:

$$
\begin{aligned}
& \mathrm{E}\left\{y_{k}(n)\right\}=A_{k} e^{j M \varphi_{1}} e^{j 2 \pi M f_{e} n}, k=0,1, \ldots, M, \\
& A_{k}:=\frac{\Gamma\left(\frac{k+M}{2}+1\right) e^{-\frac{\gamma_{1}}{2}} \rho_{1}^{M}}{\Gamma(M+1) \sigma_{1}^{M-k}} \Phi\left(\frac{k+M}{2}+1, M+1, \frac{\gamma_{1}}{2}\right),
\end{aligned}
$$

with $\sigma_{1}^{2}:=\sigma_{\mu}^{2}+\sigma_{v}^{2}$ and $\gamma_{1}:=2 \rho_{1}^{2} / \sigma_{1}^{2}$. Hence, $y_{k}(n)$ can still be viewed as a constant amplitude harmonic embedded in additive noise $v_{k}(n)=y_{k}(n)-\mathrm{E}\left\{y_{k}(n)\right\}$, and the consistency of estimators (4) hold true in the presence of flat Riceanfading channels. However, we should notice that due to the fading effect, $v_{k}(n)$ is not white any more, but a zero-mean colored stationary process, whose autocorrelation and spectral density are defined as $r_{v_{k}}(\tau):=\mathrm{E}\left\{v_{k}^{*}(n) v_{k}(n+\tau)\right\}$ and $S_{v_{k}}(f):=\sum_{\tau} r_{v_{k}}(\tau) \exp (-j 2 \pi f \tau)$, respectively. Establishing the asymptotic variance of estimators (4) in flat Riceanfading channels for any $k$ is generally, if not impossible, at least very complicated for $k=0, \ldots, M-1$. In the special case $k=M, v_{M}(n)$ is a circular noise process. Therefore, the asymptotic variance of (4) is now given by [11]:

$$
\operatorname{avar}\left(\hat{f}_{e}^{(M)}\right)=\frac{6 S_{v_{M}}\left(M f_{e}\right)}{4 \pi^{2} M^{2}\left|A_{M}\right|^{2}}
$$

The calculation of the power spectral density $S_{v_{M}}(\cdot)$ is tractable and is briefly detailed next. Let us first define the following variables:

$$
\begin{aligned}
c_{v}^{(k)} & :=\mathrm{E}\left\{|v(n)|^{2 k}\right\}=\left(\frac{\sigma_{v}^{2}}{2}\right)^{k} \frac{1}{2^{k} k !} \sum_{l=0}^{k}\left(\begin{array}{c}
k \\
l
\end{array}\right)^{2}(2 l) !(2 k-2 l) ! \\
c_{\mu}^{(k)} & :=\mathrm{E}\left\{|\mu(n)-\mathrm{E}\{\mu(n)\}|^{2 k}\right\} \\
& =\left(\frac{\sigma_{\mu}^{2}}{2}\right)^{k} \frac{1}{2^{k} k !} \sum_{l=0}^{k}\left(\begin{array}{c}
k \\
l
\end{array}\right)^{2}(2 l) !(2 k-2 l) !, \\
r_{\mu}^{(k)} & :=\mathrm{E}\left\{|\mu(n)|^{2 k}\right\}=\rho_{1}^{2 k}+\sum_{l=1}^{k}\left(\begin{array}{c}
k \\
l
\end{array}\right)^{2} \rho_{1}^{2 k-2 l} c_{\mu}^{(l)} .
\end{aligned}
$$

Some direct calculations lead to the following expression:

$$
\begin{aligned}
S_{v_{M}}\left(M f_{e}\right)= & \sum_{\tau} \sum_{k=1}^{M}\left(\begin{array}{c}
M \\
k
\end{array}\right)^{2} \rho_{1}^{2 M-2 k} c_{\mu}^{(k)} J_{0}^{k}\left(2 \pi f_{d} \tau\right) \\
& +\sum_{k=1}^{M}\left(\begin{array}{c}
M \\
k
\end{array}\right)^{2} c_{v}^{(k)} r_{\mu}^{(M-k)}
\end{aligned}
$$

\section{SIMULATION RESULTS}

In this section, we study thoroughly the performance of estimators (4) and (19) using computer simulations. Experimental mean-square error (MSE) results of (4) and (19) will be compared with the theoretical asymptotic bounds, too. The experimental results are obtained by performing a number of 200
Monte Carlo trials and all the simulations are performed assuming the frequency offset $F_{e} T=0.011$.

In this section, we also compare the asymptotic performance of proposed estimators w.r.t. MCRB, which can be expressed as $\operatorname{MCRB}\left(\hat{f}_{e}\right)=6 \sigma_{v}^{2} /\left(4 \pi^{2} N^{3}\right)$ [9, eq. (2.4.23)].

Experiment 1-Asymptotic variances of estimators (4) and (19) w.r.t. SNR: Figs. 2-3 illustrate the theoretical asymptotic variances of estimators (4) and (19) and the MCRB versus SNR. Fig. 2 depicts the asymptotic variances (12) and (26) for a BPSK modulation, assuming the number of samples $N=50$. Fig. 3 shows the performance loss (i.e., $\left.-10 \log _{10}\left[\operatorname{avar}\left(\hat{f}_{e}^{(k)}\right) /\left(N^{3} \cdot \operatorname{MCRB}\left(\hat{f}_{e}\right)\right)\right]\right)$ w.r.t. MCRB versus SNR, assuming a QPSK modulation. It can be seen that the proposed estimators exhibit good accuracy. At high SNR they coincide with the MCRB. In low SNR range, we can improve the performance of estimators (4) by adopting low order estimators ( $k=1$ and 2). Although BLUE has the best performance in the entire SNR range, the improvement is minor.

Experiment 2-Comparison of MSE of estimators (4) and (19) with the theoretical bounds: In Figs. 4 and 5, the theoretical bounds (12) and (26) are compared with experimental MSEs of the FO-estimators (4) and (19), respectively. The results are plotted versus SNR, assuming $N=50$, QPSK and BPSK modulations, respectively. Figs. 4 and 5 show that for medium and high SNR, the experimental results are well predicted by the asymptotic bounds derived in Section III, and the proposed frequency estimators provide very good frequency estimates even when a reduced number of samples is used $(N=50)$. This shows the potential of these estimators for fast synchronization of burst transmissions.

Experiment 3-Frequency offset estimators in the presence of flat Ricean-fading channel: In this experiment, we will see that the proposed frequency offset estimators perform well in the presence of Ricean-fading effects. In Fig. 6, the asymptotic variances (31) are plotted versus SNR. We assume that the Ricean-fading process has a normalized energy (i.e., $\mathrm{E}\left\{|\mu(n)|^{2}\right\}=1$ ) with Ricean factor $\kappa:=\rho_{1}^{2} / \sigma_{\mu}^{2}=1$. The Doppler spread $f_{d}$ is chosen as $0.005,0.05$ and 0.5 , respectively. The transmitted symbol is BPSK and the number of samples is chosen as $N=200$. In Fig. 6, the MSE of estimator (4) with $k=2$ and $f_{d}=0.005$ is also plotted. From Fig. 6, it turns out that although there exists an error floor due to the random fading effects, the accuracy of the proposed frequency offset estimators is still satisfying at medium and high SNRs.

Experiment 4-Frequency offset estimators in the presence of timing error: Until now, we assumed a perfect timing reference at receiver. The simulation results presented in Fig. 7 illustrate that estimators (4) are robust to timing errors. In this simulation, we assume that there is a normalized timing-error $\epsilon T=0.1$, the transmit and receive filters are square-root raised cosine filters with roll-off factor $\beta=0.5$. The symbol modulation is BPSK and the number of samples is chosen as $N=50$, 200 and 300, respectively.

\section{CONCLUSIONS}

In this paper, we have introduced and analyzed a family of non-data aided feedforward carrier frequency offset estimators. The closed-form expressions of the asymptotic variances are derived and it is shown that the proposed estimators exhibit high convergence rate and good accuracy. These estimators 
can deal with large frequency offsets $( \pm 1 / 2 M$ of the symbol rate). In addition, a best linear unbiased estimator is introduced and its asymptotic variance is established, too.

Acknowledgement: This work was supported by the NSF Career Award No. CCR-0092901.

\section{REFERENCES}

[1] M. Abramowitz and I. A. Stegun, Eds., Handbook of Mathematical Functions, Washington, DC: National Bureau of Standards, 1964.

[2] S. Bellini, C. Molinari and G. Tartara "Digital frequency estimation in burst mode QPSK transmission," IEEE Trans. Communications, vol. 38, no. 7, pp. 959-961, July 1990.

[3] S. Bellini, "Frequency estimators for M-PSK operating at one sample per symbol," Conf. Rec., GLOBECOM'94, San Francisco, 1994.

[4] J. C.-I. Chuang and N. R. Sollenberger, "Burst coherent demodulation with combined symbol timing, frequency offset estimation, and diversity selection," IEEE Trans. Communications, vol. 39, no. 7, pp. 1157-1164, 1991.

[5] F. Classen, H. Meyr, and P. Sehier, "Maximum likelihood open loop carrier synchronizer for digital radio," Conf. Rec., ICC'93, Geneva, 1993.

[6] M. Ghogho and A. Swami, "Non-efficiency of the non-linear least squares estimator of polynomial phase signals in colored noise," Conf. Rec., Asilomar'98, Pacific Grove, 1998.

[7] I. S. Gradshteyn and I. M. Ryzhik, Table of Integrals, Series, and Products, New York: Academic, 1965.

[8] F. Mazzenga and G. E. Corazza, "Blind least-squares estimation of carrier phase, Doppler shift, and Doppler rate for M-PSK burst transmission," IEEE Communications Letters, vol. 2, no. 3, pp. 73-75, 1998.

[9] U. Mengali and A. N. D' Andrea, Synchronization Techniques for Digital Receivers, Plenum Press, New York, 1997.

[10] B. E. Paden, "A matched nonlinearity for phase estimation of a PSKmodulated carrier," IEEE Trans. Information Theory, vol. 32, no. 3, pp. 419-422, May 1986.

[11] P. Stoica and R. Moses, Introduction to Spectral Analysis, Prentice-Hall, 1997.

[12] A. J. Viterbi and A. M. Viterbi, "Nonlinear estimation of PSK-modulated carrier phase with application to burst digital transmissions," IEEE Trans. Information Theory, vol. 29, no. 4, pp. 543-551, July 1983.

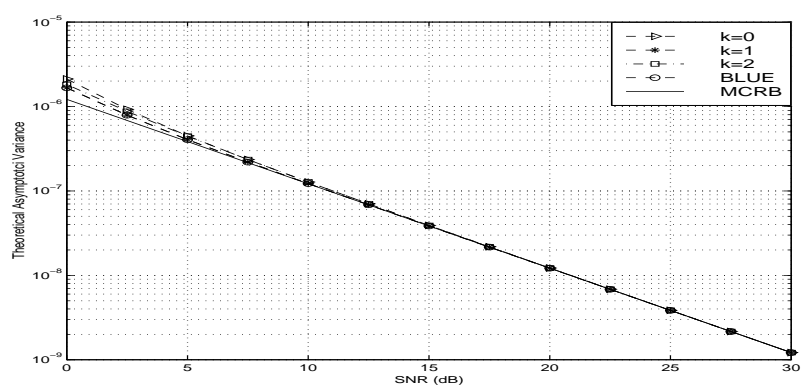

Fig. 2. Theoretical performance versus SNR for BPSK modulation.

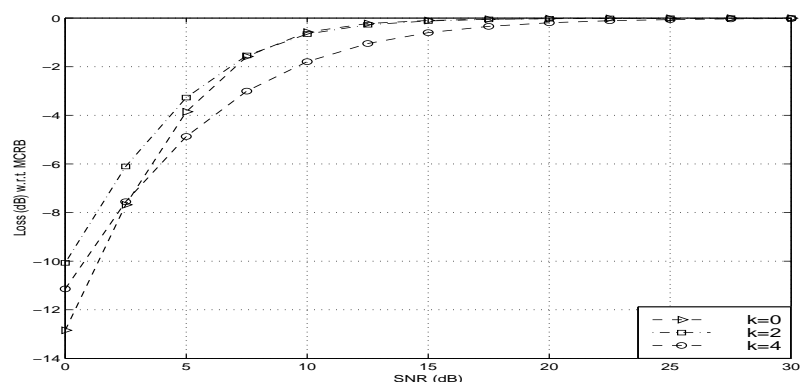

Fig. 3. Performance loss w.r.t. MCRB versus SNR for QPSK modulation.

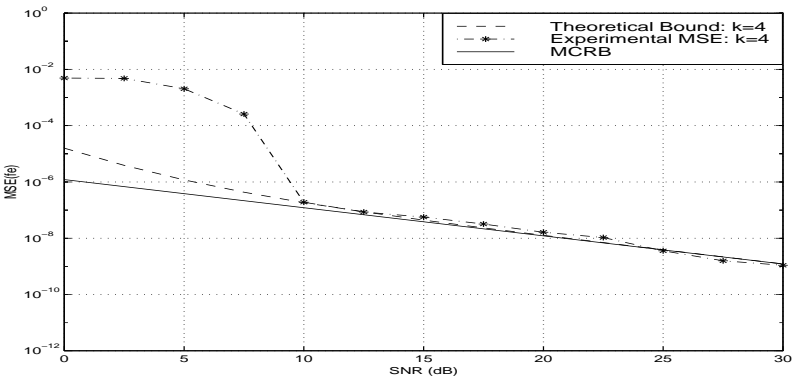

Fig. 4. MSEs of $\widehat{f}_{e}$ versus SNR for QPSK modulation (zero-padding 1024).

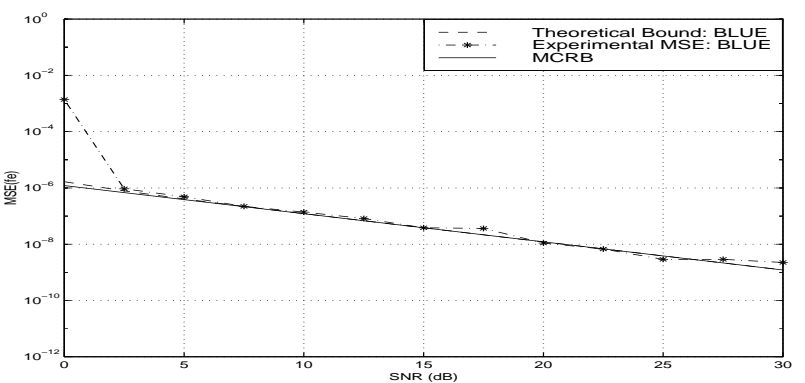

Fig. 5. MSEs of $\widehat{f}_{e}$ versus SNR for BPSK modulation (zero-padding 2048).

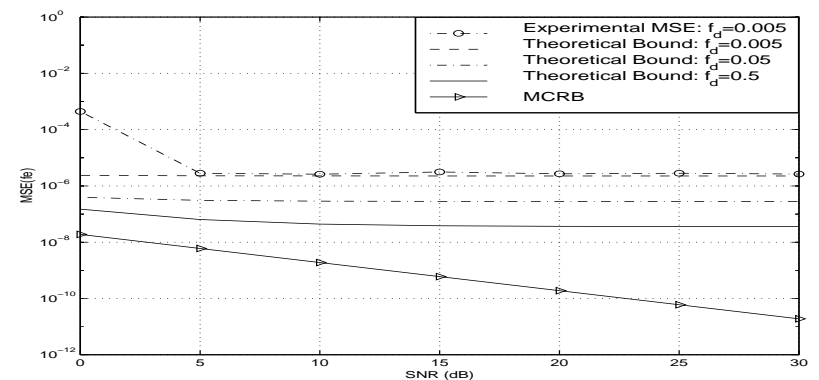

Fig. 6. MSEs of $\widehat{f}_{e}$ versus SNR for BPSK modulation in the presence of a flat Ricean-fading channel (zero-padding 1024)

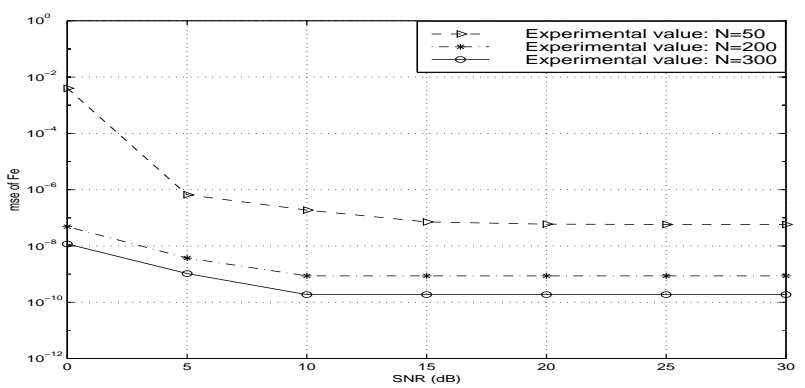

Fig. 7. MSEs of $\widehat{f}_{e}$ versus SNR for BPSK modulation in the presence of timing error $(N=50$ : zero-padding $1024, N=200$ : zero-padding 1224 , $N=300$ : zero-padding 2048) 